\title{
Prospects for the Use of Spinach (Spinacia oleracea L.) Containing Phytoecdysteroids and Polyphenols
}

\author{
Bokov DO*, Sidorova YuS, Mazo VK, Bessonov VV
}

\section{Bokov DO*, Sidorova YuS, Mazo VK, Bessonov VV}

Federal Research Center of Nutrition, Biotechnology and Food Safety, 2/14 Ustyinsky pr., Moscow, 109240, RUSSIAN FEDERATION.

\section{Correspondence}

\section{Bokov DO}

Federal Research Center of Nutrition, Biotechnology and Food Safety, 2/14 Ustyinsky pr., Moscow, 109240, RUSSIAN FEDERATION.

\section{E-mail: fmmsu@mail.ru}

History

- Submission Date: 03-12-2019;

- Review completed: 30-12-2019;

- Accepted Date: 30-12-2019.

DOI : 10.5530/pj.2020.12.38

\section{Article Available online}

http://www.phcogj.com/v12/i2

\section{Copyright}

(C) 2020 Phcogj.Com. This is an openaccess article distributed under the terms of the Creative Commons Attribution 4.0 International license.

\begin{abstract}
Background: Phytoadaptogens, biologically active compounds increasing the nonspecific resistance of the human organism, are well known for the prevention and correction of stressful conditions. Phytoadaptogens group includes phytoecdysteroids and polyphenols, that are characterized by the multiplicity of pharmacological effects in combination with the low toxicity. According to literature data, spinach (Spinacia oleracea L.) is a promising source of these compounds. This work aims to systematize data on the chemical composition of biologically active compounds of spinach, that determine its adaptogenic properties and concentration methods in the spinach processing for use in specialized foods and dietary supplements. Materials and Methods: Manifold electronic search engines, electronic databases, and libraries such as Google, Google scholar, Crossref, Indian Science Abstracts, Emerging Sources Citation Index, e-Library, Scopus, Web of Science, Pubmed, Chemical Abstracts, Index Copernicus, scientific literature had been searched and data obtained. Results: Botanical characteristics of spinach, main cultivation conditions, the latest data on the chemical composition of raw spinach material cultivars and extracts based on it are presented in this study. Schemes for the obtaining of products enriched with polyphenols and ecdysteroids are considered, and ways of proper purification are mentioned. Conclusion: It is recommended to introduce spinach into the diet of healthy people in order to increase the functional reserves of a person during periods of hypovitaminosis, overwork, intense physical exertion, and also to compensate for the adverse effects of external factors. Spinach extracts containing phytoecdysteroids (20-hydroxyecdysone) and polyphenols (flavonoids) can be used as a prophylactic to overcome the negative effects of stress, accelerate recovery after strong physical and mental stress, particularly for people with extreme occupations, athletes, and those who are engaged in hard physical labor.
\end{abstract}

Key words: Spinach leaves, Spinacia oleracea, Phytoecdysteroids, 20-hydroxyecdysone, Polyphenols, Flavonoids.

\section{INTRODUCTION}

It is known that modern man is exposed to stress factors of different nature, strength, and duration. The stress hormone cortisol takes part in the stress regulation; it mobilizes metabolic processes in the body in response to fatigue, physical exertion, starvation, fear, and other emergency stressful situations. These metabolic processes are the fission of proteins (including muscle), the start of gluconeogenesis in the liver, increase blood glucose levels, fat accumulation. The stress of low intensity and duration performs a protective effect and helps the human body overcome challenges to stressful effects. In this case, after the stress load is removed, the level of cortisol returns to the base level. In case of chronic stress, under the influence of constantly increased cortisol level, thyroid function is suppressed, blood pressure rises, hyperglycemia occurs, immunity decreases, the amount of abdominal fat increases with normal body weight, bone density decreases. This can lead to various diseases, such as diabetes, myocardial infarction, stroke, atherosclerosis. Many well-known drugs aimed at treating particular cardiovascular diseases or metabolic disorders do not eliminate their main cause due to chronic stress. ${ }^{1,2}$ Currently, much attention is paid to anti-stress phytoregulation using medicinal plants, that are effective not only for the treatment of various diseases but also as a method of normalizing the physiological functions and psychophysiological state of healthy people. Their effectiveness has been shown to adapt the body in situations of both acute stress and prolonged exposure to stress factors, to improve performance and accelerate recovery after exertion.

For the prevention and correction of stressful conditions, including fatigue, asthenia, decreased performance, adaptogens are used - biologically active compounds (BAC) mainly of plant origin, that increase the nonspecific resistance of the body to adverse stressful effects. Adaptogens act on the hormonal regulation, optimizing the secretion of cortisol and providing a tonic and stimulating effect on the nervous system and the whole human body. They are effective both at an early stage of stress and at a stage of stress exhaustion, activating metabolic processes. Adaptogens are also characterized by antioxidant action and protect cellular structures against free radicals. They possess an immunostimulating effect, increase 
physical and mental performance, resistance to various physiological and psychological stress factors, reduce adaptation time for them. Phytoadaptogens are found not only in medicinal plants but also in food plants. So, they can play the role of minor components of food. The known adaptogen sources are tinctures and drugs produced from ginseng, eleutherococcus, aralia, lemon, rhodiola and some other plants, but they have contraindications for insomnia, increased nervous irritability and hypertension. ${ }^{3-6}$

Drugs and biologically active additives based on plants containing phytoecdysteroids are devoid of these drawbacks. ${ }^{7,8}$ Phytoecdysteroids - polyhydroxylated sterols, that are structural analogs of insect molting hormones. Phytoecdysteroids are found in many species of wild plants. Phytoecdysteroids are not toxic to mammals. ${ }^{9}$ The following pharmacological effects were established for this BAC group: actoprotective, ${ }^{10}$ stress and hematoprotective, ${ }^{11}$ anti-radiation, ${ }^{12}$ antidiabetic, ${ }^{13}$ anti-atherosclerotic and anti-ischemic. ${ }^{14}$ The multiplicity of pharmacological effects in combination with the low toxicity of one of the most studied phytoecdysteroids 20-hydroxyecdysone (20E) allows its use in pharmaceuticals and dietary food supplements (DFS), especially in the nutrition of athletes. $20 \mathrm{E}$ can be isolated from medicinal plants such as safflower leuzea (Rhaponticum carthamoides (Willd.) Iljin), plumeless saw-wort (Serratula coronata L.), from edible garden spinach (Spinacia oleracea L.) and also quinoa beans (Chenopodium quinoa Willd.).

The concentration of phytoecdysteroids in spinach is 1-2 orders of value lower than in medicinal plants; S. oleracea leaves contain about $0.01 \%$ 20E per fresh raw material weight. Accordingly, the development of methods for concentrating and isolating phytoecdysteroids from plant materials are relevant.

This work aims to systematize data on the chemical composition of biologically active compounds of spinach, that determine its adaptogenic properties and concentration methods in the spinach processing for use in specialized foods and dietary supplements.

\section{MATERIALS AND METHODS}

Manifold electronic search engines, electronic databases and libraries such as Google, Google scholar, Crossref, Indian Science Abstracts, Emerging Sources Citation Index, e-Library, Scopus, Web of Science, Pubmed, Chemical Abstracts, Index Copernicus, scientific literature had been searched and data obtained.

\section{RESULTS AND DISCUSSION}

It is recommended to introduce spinach into the diet of healthy people in order to increase the functional reserves of a person during periods of hypovitaminosis, during overwork, during intense physical exertion, and also to compensate for the adverse effects of external factors (for example, low temperatures, suboptimal light conditions, increased background radiation) and the production environment (ionizing radiation, harmful chemical factors), for the regulation of carbohydrate and lipid metabolism for people with type 2 diabetes, suffering from coronary heart disease, different forms of anemia, as well as with weakening of vision and memory in the elderly. At the same time, spinach contains a large number of oxalates, which are contraindicated for people suffering from or predisposed to kidney stones, rheumatism, and gout.

One cup of raw uncut S. oleracea leaves weighing $30 \mathrm{~g}$ contains about 200 $\mathrm{mg}$ of oxalate. With a permissible oxalates daily norm of $50 \mathrm{mg}$ per day, a person suffering from these diseases can consume only 0.25 cups (or 6 g) of raw S. oleracea per day, which is not enough to obtain ecdysteroids and other micronutrients in quantities necessary for manifestation their metabolic effects. When the content of 20-hydroxyecdysone in the $S$. oleracea fresh leaves is $0.02-0.04 \%$. People suffering from kidney stone disease and with other contraindications for taking oxalates will receive approximately $1.0-2.0 \mathrm{mg}$ of ecdysteroids with a permitted portion of spinach in a quarter cup.

The minimum daily dose of ecdysteroids should be from 5 to $30 \mathrm{mg}$ to manifest its therapeutic and prophylactic effects concerning lipid and carbohydrate metabolism disorders, and to compensate for the metabolic syndrome. And for people who are engaged in hard physical labor and experience harmful production factors, such as increased radiation background, the effect of ecotoxicants, as well as for athletes, these doses should be even higher - up to 50-100 mg per day. As already noted, phytomass of $S$. oleracea contains an insignificant amount of phytoecdysteroids, therefore the pharmacological value of products obtained traditionally is insufficient due to the low content of phytoecdysteroids in it. For the same reason, the isolation of individual phytoecdysteroids from spinach is not economically viable.

S. oleracea - an annual herbaceous plant from the cinnamon family (Chenopodiaceae Vent.). This family names Amaranth (Amaranthaceae) in the more modern classification. Spinach is cultivated in temperate zones, particularly in Russia.

In the phase of industrial ripeness, it forms a basal rosette of leaves, and then an erect stem $50-90 \mathrm{~cm}$ high. The first true leaves are formed on the 4-11th day after seedlings. S. oleracea leaves arrangement is alternate, but the first 4-6 leaves do not appear sequentially one at a time, but in pairs (similar to plants with the opposite arrangement of leaves). With further growth of the stem, this arrangement is violated, and the leaves are arranged in a spiral. With the transition to the flower stalk active growth, the quality of the leaves rosette deteriorates: they become rough, the lower ones, due to the beginning of natural dying off, turn yellow and dry. The plant is cross-pollinated, often dioecious. In most varieties, female and male plants develop in approximately equal proportions, but in some varieties, there are monoecious forms. Female specimens are usually leafier. They begin to stalk later than male ones. Their growing season is longer. The flowering of all plants begins on the 15-35th day. It depends on varietal characteristics, as well as on soil and weather and climate conditions. At the same time, male plants usually bloom earlier than female ones. Female flowers located in leaf axils in the form of glomeruli or on small pedicels. They can be fertilized immediately after the appearance of stigmas and retain this ability for up to two weeks. Anther male flowers with a four-leaf pericarp and four stamens, collected in a cone-paniculate inflorescence, consistently ripen, swell, turn yellow, and sprinkle small flying pollen that is easily tolerated by the wind. Fruit - a rounded nut, smooth or with spiky outgrowths.

Spinach is cold-resistant; its seeds germinate at a temperature of 3-4 ${ }^{\circ}$ C. Seedlings and young plants can tolerate frosts of $-8{ }^{\circ} \mathrm{C}$. The leaves restore turgor after the termination the action of the low temperature. With longer frosts (up to $-10{ }^{\circ} \mathrm{C}$ ), brown leaves of dying tissues are observed on leaves after thawing (usually, differences in varieties are not observed). The most favorable temperature for the growth and development of $S$. oleracea is $15-18{ }^{\circ} \mathrm{C}$. The higher temperature, especially if it is combined with a lack of moisture in the soil and dry air, contributes to the rapid formation of the stem. With these parameters the product quality and productivity decrease. Spinach is a long day plant. In the summer, it quickly goes into the stalking phase. With a short (10-12-hour) day, S. oleracea leaves intensely grow and large rosette forms. It was established that the reaction of $S$. oleracea to the length of day and temperature conditions is associated with the early ripening of the variety. Early ripening varieties are characterized by rapid adaptation to a short day and low temperatures. For the later ripening varieties, respectively, to a long day and elevated temperature. The plant is undemanding to lighting, but with insufficient light, the content of ascorbic acid in the leaves decreases. ${ }^{15,16}$ 
S. oleracea leaves are mainly used in food (Figure 1); leaves contain protein, sugars, ascorbic acid, B vitamins, carotenoids (lutein, violaxanthin, $\beta$-carotene and 9'-(Z)-neoxanhin), polyphenols, organic acids (oxalic acid and others), phytoecdysteroids (the main component of 20-hydroxyecdysone), chlorophyll, mineral salts (iron, magnesium, potassium, phosphorus, sodium, calcium, iodine). S. oleracea flavonoid profile is represented by the following compounds: apigenin, jaceidin, kaempferol, luteolin, myricetin, patuletin, querecetin, spinacetin, 4'-glucuronide, 5,4'-dihydroxy3.3'-dimethoxy-6: 7-methylene dioxyflavone-4'-glucuronide, 3,5,7,3', 4'pentahydroxi-6-methoxiflavone, 5,3 ', 4'-trihydroxy-3-methoxy-6: 7-methylenedioxyflavone-4'-glucuronide, $\quad 5, \quad 4$ '-dihydroxi-3,3'dimithoxi-6,7-methylene-dioxyl-flavone $\left(\mathrm{C}_{18} \mathrm{H}_{14} \mathrm{O}_{8}\right) . \quad$ S. oleracea phenolic compounds include ferulic acid, para-coumaric acid, orthocoumaric acid. S. oleracea leaves are consumed boiled (in soups and as a side dish for second courses) and raw (in salads). They retain their valuable nutritional qualities in canned and freshly frozen form. The leaves can also be dried and used in powder form as a seasoning for various dishes after grinding. Juice from fresh leaves of $S$. oleracea is used. ${ }^{17-23}$

There are a significant number of spinach cultivars, some of them were carefully investigated. In the work ${ }^{24}$ a study of the polyphenols content in S. oleracea "Marquise" cultivar was carried out. An aqueous extract was obtained from raw material; the total content of polyphenols in the extract was determined using spectrophotometric and chromatographic methods; the total flavonoids content in the $S$. oleracea aqueous extract was $36.60 \mathrm{mg} / \mathrm{g}$. Moreover, apiin (16.11 mg/g), diosmethine-7apiosyl-(1->2)-glucoside (the total amount of two isomers $5.63 \mathrm{mg} / \mathrm{g}$ ) were contained in the greatest quantities. Proanthocyanidins and catechins were detected in trace amounts $(<50 \mathrm{mg} / \mathrm{kg}$ and $<10 \mathrm{mg} /$ $\mathrm{kg}$, respectively). These data differ from the results of other studies. According to them, spinach contains quercetin and its derivatives in the greatest amount (total content of $119 \mathrm{mg}$ per $100 \mathrm{~g}$ of fresh material), as well as 5,4'-dihydroxy-3,3'-dimethoxy-6, 7 a-methylenedioxyflavone 4'-O-glucuronide (37 mg / $100 \mathrm{~g}$ ). According to published data, a high apiin content is more characteristic of parsley (up to $11.89 \mathrm{mg} / 100 \mathrm{~g}$ ). The difference in the polyphenolic composition of the obtained extract from the previously obtained data can be explained by the features of $S$. oleracea "Marquise" cultivar, as well as the extraction conditions. Based on the obtained data, it can be concluded that spinach "Marquise" cultivar is a food raw material with a high content of polyphenols, especially apiin flavonoid and can be used in specialized products as a source of biologically active substances. ${ }^{24}$

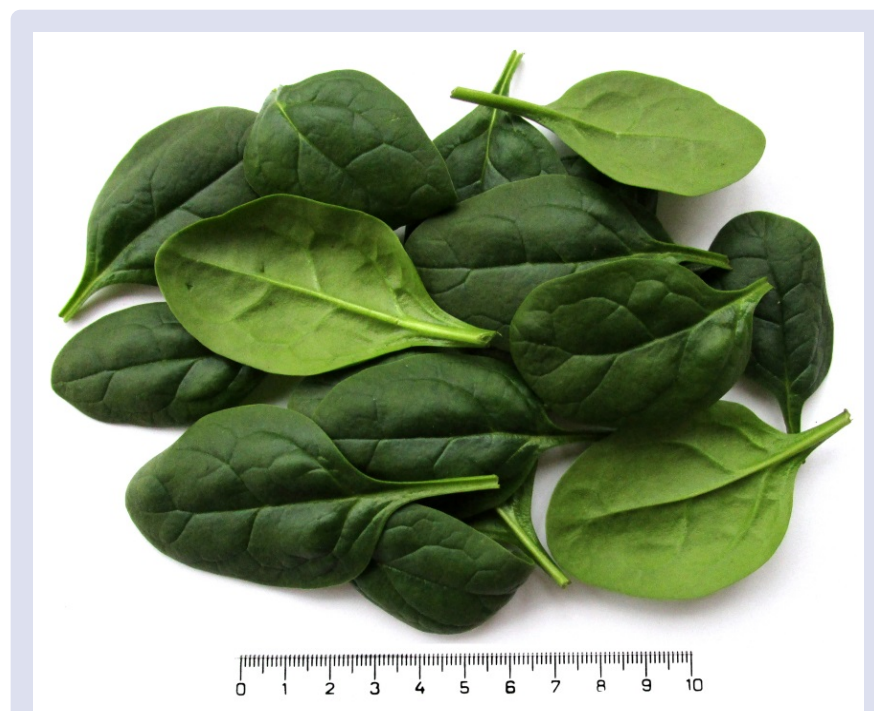

Figure 1: Spinach leaves.
In addition to leaves, $S$. oleracea seeds are a valuable raw material source. It is noteworthy that the content of oxalic acid in the $S$. oleracea seeds is significantly lower than in the leaves. The content of phytoecdysteroids is significantly higher (from 0.5 to $4 \mathrm{mg}$ per 1 g). However, the extraction of phytoecdysteroids from seeds can be complicated by the presence of cellulose, hemicellulose and pectin polysaccharides in the cell walls. Different varieties of $S$. oleracea seeds were screened by determination of the $20 \mathrm{E}$ and oxalic acid content. $S$. oleracea seeds cultivars ("Viking", "Godri", "Mariska", "Victoria", "Nafis", "Stoik", "Zastolny”, "Marquise”, "Fat-leaved”, "Matador") were provided by the All-Russian Research Institute of Selection and Seed Production were selected vegetable crops. The extraction was carried out with hot water (feed-extractant ratio, 1:20); the supernatant was separated by centrifugation, freeze-dried. The content of phytoecdysteroids was determined by the HPLC-UV method, "Marquise", "Victoria" (about 3 $\mathrm{mg} 20 \mathrm{E} / \mathrm{g}$ ) and "Matador", "Zastolny", "Fat-leaved" (about $2.5 \mathrm{mg}$ $20 \mathrm{E} / 1 \mathrm{~g}$ ) are the most promising varieties of $S$. oleracea seeds in view of phytoecdysteroids extraction. The protein content in the S. oleracea dry seed extract was determined by the Kjeldahl method (13.8 \%). The average concentration of oxalic acid in extracts from $S$. oleracea seeds was about $4 \mathrm{mg} / \mathrm{g}$ in dry extract (determined by titration with potassium permanganate); it indicates that $S$. oleracea seeds are promising source of natural phytoecdysteroids. The possible usage of $S$. oleracea seeds dry extracts for preparation of dietary supplements and specialized products requires additional toxicological and hygienic studies. ${ }^{25}$

\section{Methods of functional extracts formulation from $\mathrm{S}$. oleracea raw material}

There are different methods of obtaining extracts from S. oleracea leaves according to the target BAS group.

To obtain a polyphenolic extract from S. oleracea leaves, edible oil is added to the aqueous extract according to patent authored by S. Duval. ${ }^{26}$ Extraction is carried out, concentrated in vacuo and the oil and water phases are separated. The resulting extract from the aqueous phase is enriched in polyphenols by more than $50 \%$. There is no accounting of phytoecdysteroids concentration in this method. The final product and oxalates are not removed from the target aqueous phase of the extract. This worsens the consumer properties of the extract and restricts its use for a wide range of people for whom the use of oxalates is contraindicated.

In another method, these disadvantages are eliminated. ${ }^{27}$ The scheme for obtaining this extract is presented in Figure 2.

This extract from S. oleracea leaves is enriched with phytoecdysteroids (mostly 20-hydroxyecdysone) and does not contain oxalates. To obtain it, the S. oleracea dried crushed leaves are extracted with water at a ratio of water: leaves $40: 1$ at a temperature of $40-90{ }^{\circ} \mathrm{C}$ for $10-90$ minutes. The resulting aqueous extract is separated from the biomass, particularly by centrifugation. The aqueous extract is subjected to ultrafiltration at room temperature to remove suspended and colloidal substances, mainly pigments and lipids. Then the low molecular weight fraction is extracted by solid-phase extraction of phytoecdysteroids using hydrophobic sorbents at a sorbent: fraction ratio of 7:1. Also the sorbent can be added to the low molecular weight fraction, at a ratio of fraction: sorbent 7.5:1 and incubated for 1 hour. Then the sorbent is washed with water. The desorption of phytoecdysteroids with related substances is carried out by known methods, for example, aqueous solutions of lower alcohols with an alcohol concentration of 5 to 80 volume percent, particularly $70 \%$ ethanol. The obtained extract is evaporated and dried, in the particular case, by freeze drying.

The content of 20-hydroxyecdysone in the dry concentrate extract varies from 1.0 to $20.0 \mathrm{mg}$ per $1 \mathrm{~g}$ of the dry preparation, depending on the volume fraction of lower alcohol in the solvent used at the 


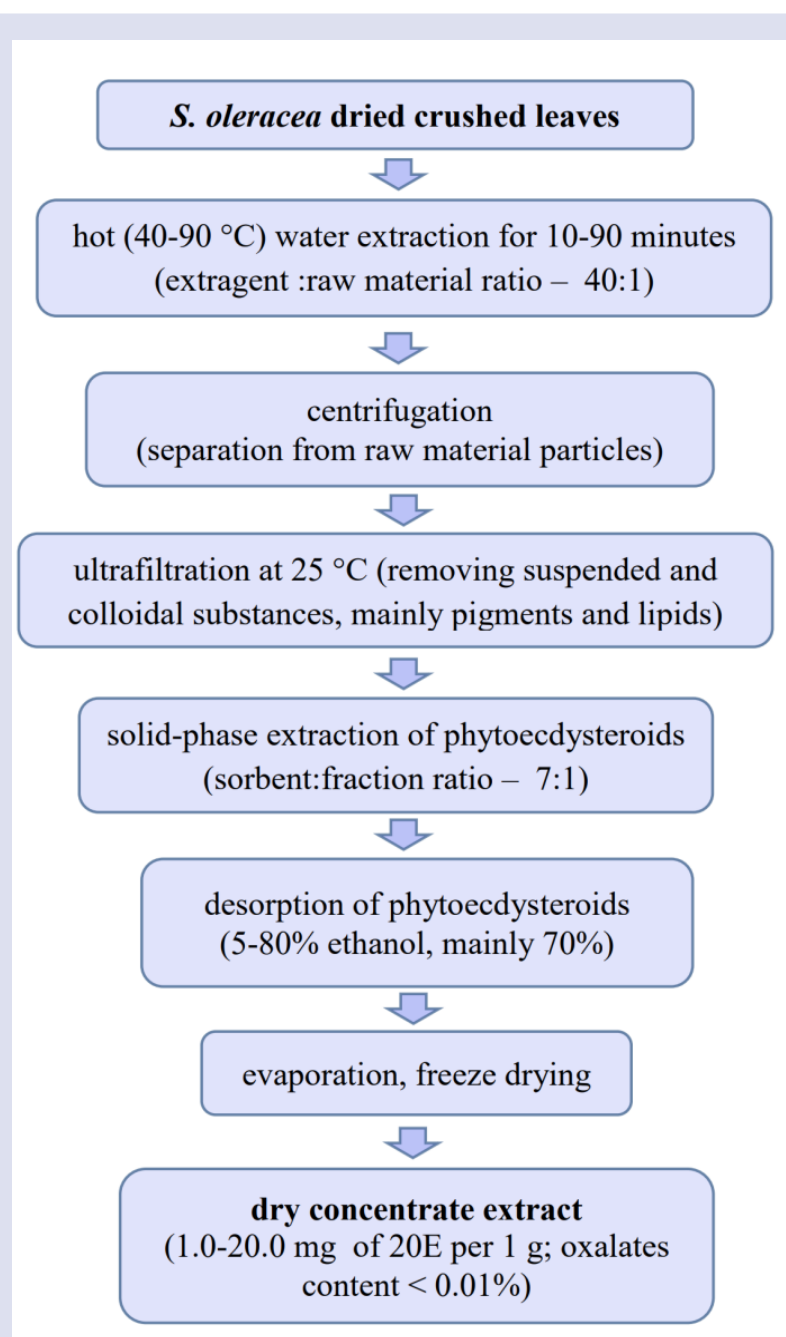

Figure 2: Scheme for obtaining dry concentrate extract enriched with phytoecdysteroids.

stage of desorption of ecdysteroids. The content of oxalates does not exceed $0.01 \%$. Thus, the researchers developed an effective way to obtain an extract from the $S$. oleracea leaves. It allows to increase the 20-hydroxyecdysone content in the dry enriched concentrate extract up to $1.0-20.0 \mathrm{mg}$ per $1 \mathrm{~g}$ of dry extract and reduce the content of oxalates to $0.01 \%$.

\section{CONCLUSION}

The chemical composition of raw spinach is considered. The main groups of biologically active compounds are phytoecdysteroids (20-hydroxyecdysone) and polyphenols (flavonoids). Methods for producing extracts enriched in polyphenolic compounds with phytoecdysteroids are searched and compared. Spinach extracts containing these groups of compounds can be used as a prophylactic to overcome the negative effects of stress, accelerate recovery after strong physical and mental stress, particularly for people with extreme occupations, athletes, and those who are engaged in hard physical labor. Also, it can be used for people that are exposed to harmful natural and industrial factors, for elderly people experiencing chronic stress.

\section{ACKNOWLEDGMENT}

The study was financially supported by the Russian Science Foundation, grant No. 19-16-00107 "New functional food ingredients of adaptogenic action for the enhancement of working capability and cognitive potential of human organism"

\section{CONFLICTS OF INTEREST}

None.

\section{REFERENCES}

1. Dilman VM. Big biological clock (Introduction to integrated medicine). Moscow Knowledge 1981;208.

2. Levin O. Phyto-adaptogens - protection against stress? Harefuah 2015;154(3):183-6.

3. Dyrdymov IV. Ginseng, Eleutherococcus (to the mechanism of biological action). Moscow 1976;184.

4. Yaremenko KV. The optimal state of the body and adaptogens. Saint-Petersburg 2007;129.

5. Martinussen I, Volodin V, Volodina S, Uleberg E. Effect of Climate on Plant Growth and Level of Adaptogenic Compounds in Maral Root (Leuzea charthamoides (Willd.) DC.), Crowned Saw-wort (Serratula coronata L.) and Roseroot (Rhodiola rosea L.).

6. Kumar GP, Anilakumar KR, Naveen S. Phytochemicals Having Neuroprotective Properties from Dietary Sources and Medicinal Herbs. Pharmacognosy Journal. 2015;7(1):1-17.

7. Volodin VV, Mataev SI. Ecdysteroid-containing plants - sources of new adaptogens. Bulletin of Biotechnology and Physico-Chemical Biology named after Yu. A. Ovchinnikov 2011;7(2):52-9.

8. Dinan L, Harmatha J, Volodin V, Lafont R. Phytoecdysteroids: diversity, biosynthesis and distribution. In Ecdysone: structures and functions. 2009 Springer, Dordrecht, 3-45.

9. Tarkowská D, Strnad M. Plant ecdysteroids: plant sterols with intriguing distributions, biological effects and relations to plant hormones. Planta. 2016;244(3):545-55

10. Patent RU No 2276991 "Tonic and actoprotective agent "Serpisten" priority from 02.09.2005, published 05.27.2006.

11. Patent RU No 2375071 "Antiaggregational and stress-limiting agent" priority of November 6, 2008, published December 10, 2009

12. Patent RU No 2236672 "Anti-radiation agent," priority of March 21, 06, published 06.20.2008.

13. Patent RU No 2337698, "Antidiabetic agent with lipid-lowering asset nostrum for the treatment and prevention of type II diabetes mellitus", priority dated 06.02.2007, published 10.11.2008

14. Patent RU No 2337701 "Hypolipidemic and anti-ischemic agent "Serpisten", priority dated 06.02.2007, published November 10, 2008.

15. GOST R 55650-2013. Group C42. National standard of the Russian Federation Fresh sorrel and spinach. Technical conditions.

16. Kamaev IN, Kamaev AN. Small-spread vegetable crops in the Stavropol Territory. Stavropol: Prince. Publishing House 1992;144.

17. Gaikwad PS, Shete RV, Otari KV. Spinacia oleracea Linn: A pharmacognostic and pharmacological overview. International Journal of Research in Ayurveda and Pharmacy (IJRAP). 2010;1(1):78-84.

18. Grevsen K, Kaack K. Quality attributes and morphological characteristics of spinach (Spinacia oleracea L.) cultivars for industrial processing. Journal of Vegetable Crop Production. 1997; 2(2):15-29.

19. Singh K, Singh DK, Singh VK. Chlorophyllin treatment against the snail Lymnaea acuminata: A new tool in fasciolosis control. Pharmacognosy Journal. 2017;9(5):594-8.

20. Chaturvedi D, Singh DK, Singh VK. Photodynamic Toxicity of Chlorophyllin against Fasciola gigantica Carrier Snail Indoplanorbis exustus in Visible Spectra Band. Pharmacognosy Journal. 2017;9(6):729-36.

21. Cheng DM, Yousef GG, Lila MA. Variation in phytoecdysteroid accumulation in seeds and shoots of Spinacia oleracea L. accessions. Hort Science. 2010;45(11);1634-8.

22. Metha D, Belemkar S. Pharmacological activity of Spinacia oleracea Linn - a complete overview. Asian Journal of Pharmaceutical Research and Development. 2014;83-93.

23. Bakrim A, Maria A, Sayah F, Lafont R, Takvorian N. Ecdysteroids in spinach (Spinacia oleracea L.): biosynthesis, transport and regulation of levels. Plant Physiology and Biochemistry. 2008;46(10):844-54.

24. Morina NS, Sidorova Yu S, Nechaev AP. The Polyphenol Content in Aqueous Extract of Spinach. Storage and processing of Agricultural Raw Materials. 2016;1:19-22.

25. Sidorova YuS, Bogachuk MN, Nastrulina AA. Screening for 20-hydroxyecdysone in various spinach seed varieties. Voprosy Pitaniia. 2014;83(S3):240.

26. Patent RU 2519640 "Method for the preparation of polyphenolic extracts from S. oleracea leaves" priority 04.05.2009, published on 06.20.2014.

27. Patent RU 262745009 "Spinach leaf extract and method for producing it" 11.2015. 


\section{GRAPHICAL ABSTRACT}

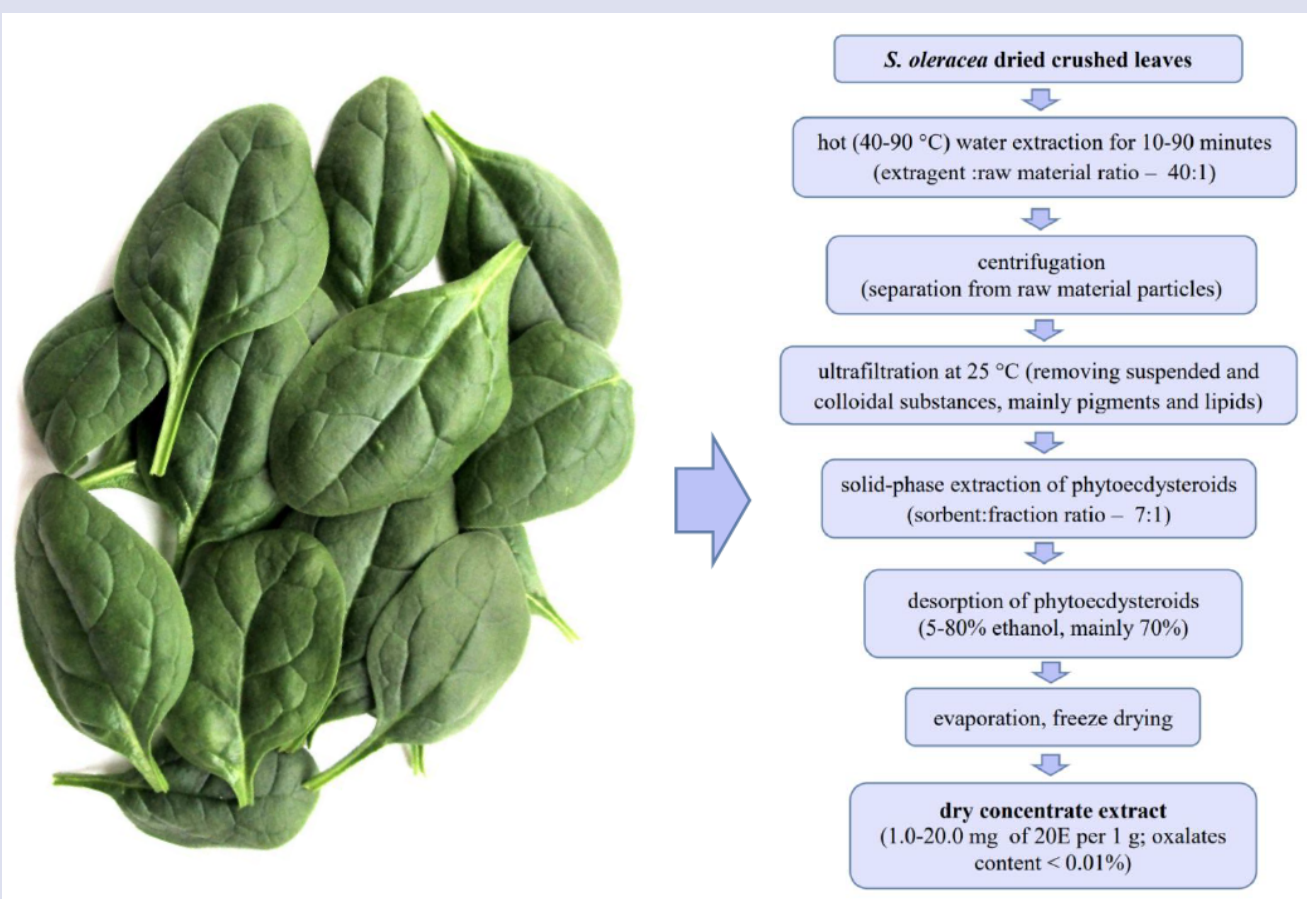

\section{ABOUT AUTHORS}

- Bokov Dmitry Olegovich - Ph.D in Pharmaceutical Sciences, researcher of laboratory of food chemistry, Federal Research Center of Nutrition, Biotechnology and Food Safety. Research Interests: food chemistry, analytical chemistry, pharmacognosy, investigation of the composition and content of biologically active compounds in crude herbal drugs using modern instrumental methods of analysis (HPLC with RID, UV, MS-MS detection, GC-MS).

- Sidorova Yuliia - PhD, senior researcher of laboratory of food biotechnology and specialized products, Federal Research Center of Nutrition, Biotechnology and Food Safety. Research Interests: food biotechnology, biomodelling, physiology, protein chemistry, plant biologically active substances, adaptation, stress, neuroscience. For her dissertation research she developed and approved the novel experimental complex approach to determination of adaptogenic properties of minor plant biologically active substances.

- Mazo Vladimir Kimovich - Professor, doctor of Biological Sciences, laboratory of food biotechnology and specialized products, Federal Research Centre of Nutrition, Biotechnology and Food Safety. Research Interests: the development of technology of functional food ingredients production for specialized food with hypoglycemic and hypolipidemic action; the development of methods of production and experimental evaluation of effectiveness and safety of essential microelements organic form; physiological and biochemical evaluation of plant adaptogens in vivo.

- Bessonov Vladimir Vladimirovich - Doctor of Biological Sciences, head of laboratory of food chemistry, Federal Research Center of Nutrition, Biotechnology and Food Safety. Research Interests: study of technological contaminants and secondary oxidation products content in highly processed dietary fats; development of a methodological and regulatory framework to ensure modern requirements for the quality of food products and the development of technologies for evaluating the effectiveness of specialized food products.

Cite this article: Bokov DO, Sidorova YuS, Mazo VK, Bessonov VV. Prospects for the Use of Spinach (Spinacia oleracea L.) Containing Phytoecdysteroids and Polyphenols. Pharmacog J. 2020;12(2):246-50. 